\title{
JUURNAL_RU
}

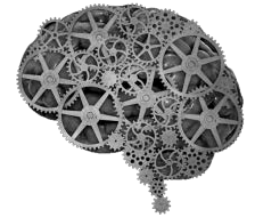

COMPANY GROUP "INTELLEKT"

\author{
Дикарев В.И. ${ }^{1}$, Березин Б.В. ${ }^{2}$, Казаков Н.П. ${ }^{2}$ \\ ${ }^{1}$ ВИКА им. А.Ф. Можайского \\ Санкт-Петербург, Россия \\ ${ }^{2}$ НИО НИИ ВСИ ВА МТО \\ Санкт-Петербург, Россия
}

doi: 10.18411/lj2016-7-2-06

idsp 000001: lj2016-16-2-06

\section{Использование радиоинтерферометров со сверхдлинными базами для исследования объектов ближнего и дальнего космоса (Лунный проект)}

В статье излагаются возможности построения высокоточной системы наблюдения и контроля за космическими объектами. Расположение одного из радиотелескопов на Луне позволит использовать радиоинтерферометры со сверхдлинными базами, что, в свою очередь, значительно повысит точностьопределения местоположения космических объектов.

Ключевые слова: радиоинтерферометры со сверхдлинными базами, космические объекты, радиотелескопы, радиосигналы.

Современные космические наблюдения с использованием радиоинтерферометров со сверхдлинными базами (РСДБ) позволяют определять различные параметры (положение источников радиоизлучений, координаты комет, астероидов, космических станций, характеристики вращения Земли и Луны и т.п.) с точностью $\lambda / \mathrm{d}$, где $\lambda$ - длина волны наблюдений, $\mathrm{a} d-$ измерительная база между двумя радиотелескопами.

В настоящее время длина волны наблюдений составляет до 7,5 мм, а длина измерительной базы ограничена диаметром Земли. Наблюдения на более 
коротких длинах волн невозможны из-за интенсивного поглощения таких волн земной атмосферой и высоких требований к аппаратуре [1].Следовательно, единственным методом увеличения точности измерений в космосе является увеличение длины измерительной базы посредством использования радиотелескопа за пределами Земли.

Предлагается использовать три радиотелескопа А,В и С, один из которых С разместить на Луне, а два других А и В - на Земле, образуя тем самым три сверхдлинные измерительные базы (d1, d2, d3), расположенные в виде треугольника (рис.1).

Радиотелескопы А,В и С синхронно направляют на исследуемый космический объект (КО), который излучает широкополосные шумоподобные или любые другие радиосигналы, например, сигналы автоматических межпланетных станций (к примеру, «Экзомарс»). Указанные радиосигналы по линиям связи ЛС1, ЛС2, ЛС3 передают в центр обработки информации (ЦОИ) [2].

Шумоподобные сигналы:

$$
\begin{gathered}
\mathrm{U} 1(\mathrm{t})=\mathrm{V} 2 \cdot \cos [\omega \mathrm{ct}+\varphi \kappa(\mathrm{t})+\varphi 1], \\
\mathrm{U} 2(\mathrm{t})=\mathrm{V} 2 \cdot \cos [\omega \mathrm{c}(\mathrm{t}-\tau 1)+\varphi \kappa(\mathrm{t}-\tau 1)+\varphi 2], \\
\mathrm{U} 3(\mathrm{t})=\mathrm{V} 3 \cdot \cos [\omega \mathrm{c}(\mathrm{t}-\tau 2)+\varphi \mathrm{Kt}-\tau 2)+\varphi 3], 0 \leq \mathrm{t} \leq \mathrm{Tc},
\end{gathered}
$$
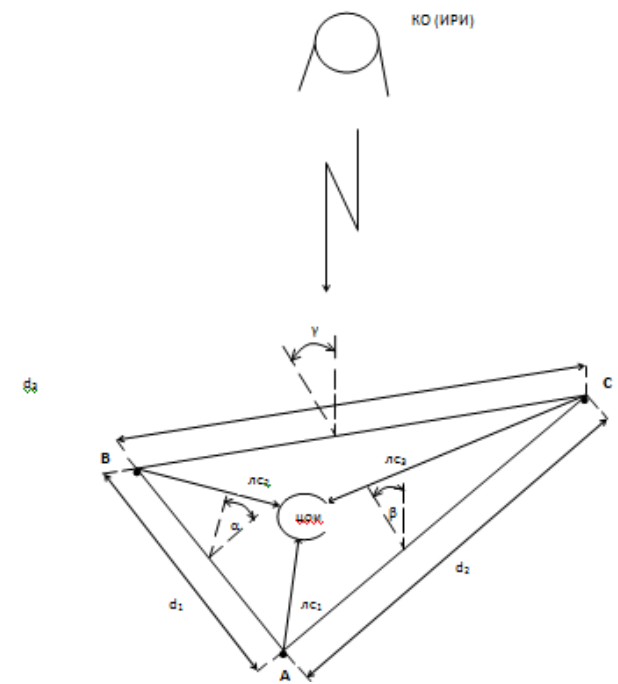

Рис.1. Геометрическая схема расположения космического объекта (КО) и радиотелескопов $A, B, C$, размещенных на Земле и Луне. 
где $\varphi \kappa(\mathrm{t})=\{0, \pi\}-$ манипулирующая составляющая фазы, отображающая закон фазовой манипуляции:

$\tau 1=\frac{\mathrm{d} 1 \sin \alpha}{c}-$ время запаздывания сигнала, приходящего на радиотелескоп В по отношению к сигналу, приходящего на радиотелескоп А;

$\tau 2=\frac{\mathrm{d} 2 \sin \beta}{c}-$ время запаздывания сигнала, приходящего на радиотелескоп С по отношению к сигналу, приходящему на радиотелескоп А;

$\tau 3=\frac{\mathrm{d} 3 \sin \gamma}{c}-$ время запаздывания сигнала, приходящего на радиотелескоп С по отношению к сигналу, приходящему на радиотелескоп В;

d1,d2,d3 - сверхдлинные измерительные базы;

$\alpha, \beta, \gamma-$ азимут, угол места и угол ориентации КОили источника радиоизлучения (ИРИ).

с выходов приемников ПРМ1,ПРМ2 и ПРМЗ поступают на удвоители фазы, а затем на делители фазы на два (рис.2). На выходах последних образуются следующие гармонические колебания соответственно:

$$
\begin{gathered}
\mathrm{U}_{4}(\mathrm{t})=\mathrm{V}_{4} \cdot \cos \left(\omega_{\mathrm{c}} \mathrm{t}+\varphi_{1}\right), \\
\mathrm{U}_{5}(\mathrm{t})=\mathrm{V}_{5} \cdot \cos \left[\omega_{\mathrm{c}}\left(\mathrm{t}-\tau_{1}\right)+\varphi_{2}\right], \\
\mathrm{U}_{6}(\mathrm{t})=\mathrm{V}_{6} \cdot \cos \left[\omega_{\mathrm{c}}\left(\mathrm{t}-\tau_{2}\right)+\varphi_{3}\right], 0 \leq \mathrm{t} \leq \mathrm{T}_{\mathrm{c}},
\end{gathered}
$$

которые выделяются узкополосными фильтрами соответственно и поступают на входы фазометров $\Phi 1$, Ф2 и ФЗ. Последние измеряют следующие разности фаз:

$$
\begin{aligned}
& \Delta \varphi_{1}=\varphi_{2}-\varphi_{1}=2 \pi \frac{\mathrm{d} 1}{\lambda} \cos \alpha, \\
& \Delta \varphi_{2}=\varphi_{3^{-}} \varphi_{1}=2 \pi \frac{\mathrm{d} 2}{\lambda} \cos \beta, \\
& \Delta \varphi_{3}=\varphi_{3}-\varphi_{2}=2 \pi \frac{\mathrm{d} 3}{\lambda} \cos \gamma,
\end{aligned}
$$

где $\lambda$-длина волны,

которые фиксируются компьютером КОМ (рис.2).

Так формируются фазовые шкалы отсчета угловых координат $\alpha, \beta$ и $\gamma$ : точные, но не однозначные. 
Принимаемые шумоподобные сигналы U1(t),U2(t) иU3(t) одновременно поступают на два входа корреляторов КОР1,КОР2 и КОРЗ. Получаемые на выходе фильтров нижних частот ФНЧ1,ФНЧ2 и ФНЧЗ

корреляционные функции $\mathrm{R} 1(\tau), \mathrm{R} 2(\tau)$ иR3( $\tau)$ имеют максимум при значении введенной регулируемой задержки:

$$
\tau_{1}=\mathrm{t}_{2}-\mathrm{t}_{1}, \tau_{2}=\mathrm{t}_{3}-\mathrm{t}_{1}, \tau_{3}=\mathrm{t}_{3}-\mathrm{t}_{2},
$$

где t1,t2ut3 - время прохождения сигналом расстояний от КО (ИРИ) до радиотелескопов А,В и С соответственно.

Максимальные значения корреляционных функций $\operatorname{R} 1(\tau), \operatorname{R} 2(\tau)$ иR3 $(\tau)$ поддерживаются с помощью экстремальных регуляторов ЭР1,ЭР2 и ЭРЗ, воздействующих на управляющие входы блоков регулируемой задержки БР31,БР32 и БРЗ3.

Шкалы БР31,БР32 и $\quad$ БР33 (указатели углов) проградуированы непосредственно в значениях угловых координат $\alpha, \beta$ и $\gamma$ КО (ИРИ):

$$
\alpha=\operatorname{arccosc} * \tau 1 / \mathrm{d} 1 ; \beta=\operatorname{arccosc} * \tau 2 / \mathrm{d} 2 ; \gamma=\operatorname{arccosc} * \tau 3 / \mathrm{d} 3 .
$$

Значения угловых координат $\alpha, \beta$ и $\gamma$ фиксируются соответствующими индикаторами и поступают в компьютер КОМ.

Так формируются временные шкалы отсчета угловых координат $\alpha, \beta$ и $\gamma$ : грубые, но однозначные.

Следует отметить, что расположение радиотелескопов А,В и С в виде треугольника продиктовано новой идеологией фазовой пеленгации КО (ИРИ)

в пространстве, которая обеспечивает определение местоположения КО (ИРИ) и его перемещения в пространстве пассивным методом. Причем для точного и однозначного определения местоположения КО (ИРИ) и его

перемещения в пространстве используются фазовые шкалы отсчета угловых координат $\alpha, \beta$ и $\gamma$ : точные, но не однозначные и временные шкалы отсчета угловых координат $\alpha, \beta$ и $\gamma$ : грубые, но однозначные, получаемые за счет корреляционной обработки принимаемых сигналов. 
Размещение одного из радиотелескопов на Луне позволяет увеличить рабочую длину измерительной базы в 60 раз для международной РСДБ - сети и почти в 90 раз для радиоинтерферометра «Квазар - КВО». В свою очередь, увеличение длины измерительной базы позволит производить позиционные наблюдения радиоисточников с точностью до 1 микросекунды дуги. Это соответствует точности 1 мм в положениях станций, за которыми ведется контроль.

Существенным основанием для создания лунного радиотелескопа являются научно-технические достижения России: в частности, единственным действующим радиотелескопом за пределами Земли является 110 - метровый космический телескоп «Радиоастрон», созданный в НПО им. Лавочкина по заказу АКЦ ФИАН [1]. Этот телескоп спроектирован для решения астрофизических задач и по ряду причин не может быть использован для исследования объектов ближнего и дальнего космоса.

В случае успеха предлагаемый лунный проект станет первым и уникальным в своем роде сооружением и позволит решать мировые научные задачи:

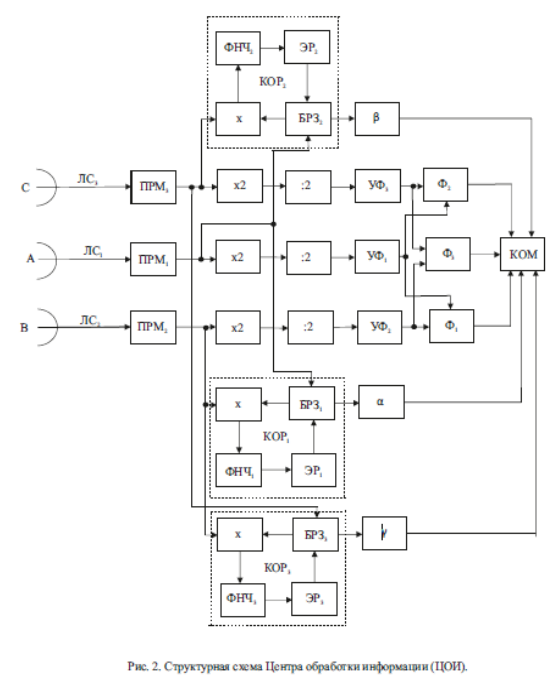

Рис.2. Структурная схема Центра обработки информаиии (ЦОИ). 
- повышение точности реализации Международной небесной системы координат (ICRF) более чем на порядок;

- точное задание ориентации группировки ГЛОНАCC в ICRF, что обеспечит высокоточную навигацию в космическом пространстве в инерциальной системе отчета;

- изучение внутреннего строения Луны из анализа высокоточной моделиее вращения;

- повышение точности эфемерид планет солнечной системы;

- проверка научных гипотез из области астрофизики и релятивистской небесной механики;

- повышение точности и однозначности определения местоположения и перемещения в пространстве спутниковой глобальной навигационной системы ГЛОНАСС и геостационарных ИСЗ-ретрансляторов, используемых в дуплексном методе сличения удаленных шкал времени, а также в службе единого времени и частоты;

- повышение точности и однозначности определения местоположения и перемещения в пространстве небесных тел, планет, метеоритов, астероидов, находящихся в ближнем и дальнем космосе;

- повышение точности и однозначности определения местоположения и перемещения в пространстве источников радиоизлучений, размещенных на различных носителях (космические аппараты, самолеты, ракеты и т.п.).

Кроме того, предлагаемый лунный проект даст толчок к разработке технологийдля:

- роботизированных миссий по возведению дистанционно управляемых прецизионных конструкций на поверхности Луны, что является новой и уникальной задачей; 
- мягкой посадки на Луну, что актуально для всей отечественной лунной программы;

- обмена (по оптическому и радиоканалу) большими объемами цифровых данных между Землей и Луной.

В целом предлагаемый лунный проект отличается новизной, оригинальностью, является перспективным и может найти широкое практическое применение при освоении человеком ближнего и дальнего космоса.

\section{Литература:}

1. Финкельштейн А.М., Ипатов А.В. и др. Радиоинтерферометрическая сеть «Квазар - КВО» - базовая система фундаментального координатно-временного обеспечения.- СПб., Труды ИПА РАН, №13, 2005.- C.104-138.

2. Дикарев В.И. Заявка на изобретение. Радиоинтерферометрический способ исследования объектов ближнего и дальнего космоса и система для его реализации. G01 S 3/72, 2016. 